resemble each other minutely in their morphological and cultural characters. May it not be that the pathogenic yeasts can be altered similarly by local conditions? It is possible that these fungi, though normally non-pathogenic, can, as in the case of the pneumococcus and diphtheria bacillus, assume at times a pathogenic róle.

A minute study of the microscopical sections of the tongue and oesophagus in sprue shows that there is a considerable reaction on the part of the host to the invasion of the superticial tissues by this fungus, and there can be little dotibt that these fungi in their downward growth are capable of exposing the taste buds and nerve terminals and of causing the ving lesious which the lingual and oesophageal symptoms of rerye suggest.

8. Wasting and anaemia-both symptoms of sprue-can be produced in rabbits by continuous intravenous injections of small doses of a broth culture of a pathogenic yeast.

9. Diarrhoea, atrophy of the lingual papillae as in spruc, digestive disturbances, and aplithous ulceration of tlie mouth are commonly found in children the subjects of a thrush infection in temperate zones.

10. It is possible that obscuie alimentary diseases of children in temperate zones - such as Gice's coeliac dianrhoea-are of the same nature as sprue in the tropics. A hypothesis of this sort would explain the occurrence of sporadic cases of sprue in temperate zones.

11. The local affection of different portions of the digestive tract with this fungus would best explain the varying clinical manifestations of sprue.

12. To maintain such a hypothesis it is necessary to stipulate for a third factor, a predisposing cause which may exist in local tropical conditions-conditions which facour a more precocious and luxuriant growth of all fingi, a matter of common observation to laboratory workers in the tropics.

\section{General Conclusions.}

Finally, as a result of my investigations I am able to put forward the following conclusions:

1. Sprue is a specific disease of tropical and subtropical countries, thongh it is possib!e that cases occasionally originate in temperate zones.

2. It is a prevalent disease in Ceylon, especially amongst the Europeans. Contrary to the opinion hitherto held, it occur's amongst the natives irrespective of race or mode of life.

3. This fact, together with the occurrence of the disease in people closely associated with one another, suggests some local influence or some communication of the specific cause from man to man.

4. Sprue is a variable disease. It may occur in a mild or in a particularly virulent form, and, in common with many other serious diseases, is prone to sudden exacerbations, remissions, and periods of latency.

5. 'There is evidence that the disease may occur as distinct clinical forms, according to the portion of the alimentary canal attacked.

6. Researches in the composition of the stools point either to a complete absence or inadequacy of the intestinal digestive ferments.

7. Researches on the blood and on the urine suggest that certain of the more important clinical features of spive are dependent on an alimentary toxaemia.

8. The pathological findings are also in favour of such a conclusion, and, if anything, point to an invasion with the thrush fungus (Monilia albicans) as being concerned in their production.

1 Rohlbrugge: Necl. Tidjschr. $v$ Gences.

政, Archiv, 1901, vol. v, No. 12, p. $394 .{ }^{2} \mathrm{~L}$
Soc. de Biol., 1908, 1 xiv, No. 21, p. 1066.

THE report presented to the annual meeting of the Automobile Association and Motor Union shows that it has 86,402 members. In addition to ordinary members, there are light car members who pay an annual subscription of 1 guinea and 32,000 motor cyclist members paying half a guinea. Mr. Joynson-Hicks, in moving the adoption of the report, said that proposals for the further taxation of motorists should be resisted. He believed that a properly made road was not damaged by motor traffic, and that, as ratepayers and taxpayers, motor users were entitled to demand that roads should be up to sufficient strength to carry the heaviest form of traffic.
THE “ CONTROLLED" USE OF

NEW TUBERCULIN IN THE TREATMENT OF PULMONARY TUBERCULOSIS.

G. V. STOCKDALE, M.B., Ch.B.Lerds, AND

RONALD IHODSON, M.B., B.C.CANTAB., M.R.C.S L.R.C.P.,

LATE hotsr-physicians at THF Hospital for coNscMption aND DISEASES OF THE CHEST, BROMPTON.

Ir is obviously absurd to say that tuberculin is of valus because two or three patients have improved under it, just as the converse is equally absurd. What we have dono here is to attempt to work on some fair system by whicl to prove the efficacy or otherwise of tuberculin, and the system we adopted was one of controls. As many cases as possible of pulmonary tuberculosis (the critcrion for which was the presence of tubercle bacilli in the sputum) were collected and paired off in such a way that a febrilc patient was paired with another febrile, whilst an afebrilo was paired with an afebrile. Then by lot it was decided which should be given tuberculin, so that there should bo no suggestion as to picking cases, for if tuberculin is to b3 given with any idea of proving its value, it is, of course, useless to administer it only in cases in which the prognosis is distinctly favourable. Further, as fresh patients were admitted into the hospital, it was decided by lot which should be given tuberculin and which should act as controls.

Although we admit that it is practically impossible to select tivo cases p:ecisely similar in degree and extent, yot we think that if a sufficiently large number of cases is observed on this plan this difficulty is to a great extent abolished.

The period over which the treatment should extend was decided as three months. This, unfortunately, is too short, yet we find that it is a period commonly chosen for tuberculin treatment in private practice, and the difficulty of keeping several patients in hospital for a longer period was very great.

The cases were classified into three groups

1. Afebrile cases.

2. Intermittently febrile cases-that is, using the torm "intermittent" in the sense that after a day or two rise the temperature would fall to normal, and then a further rise would be recorded.

3. Febrile cases.

A temperature of $99^{\circ} \mathrm{F}$. was our standard; anything over that was considered to be febrile, and the patients were consequently liept in bed. If, however, at the end of the second day following this the temperature was below $99^{\circ} \mathrm{F}$. they were allowed up for two hours, this period being gradually increased day by day as long as therc was absence of fever. Many of the patients remained febrile as long as a month, and then would pass into an intermittently febrile or afebrile condition, but to overcome this difficulty the original classification was adhered to. Such cases as were severely ill, and thoso complicated with pleural effusion which masked the signs, by severe recent haemoptysis so that they could not be examined fairly, and extensive laryngitis which obscured the pulmonary sounds, were not included in our series.

\section{Method of Administration.}

The tuberculin selected to be used was the new tubcrculin T.R., and it was decided to give minute doses, increasing the doses gradually every second or third day at first and later at longer intervals in the case of the afebrile patients, while the febrile patients received a dose every fifth or seventh day. The initial dose in the former case was 1 one hundred thousandth milligram, and was increased to $2,3,5,8$ and 12 one hundred thousandths milligram, and then again about three-fourths as much again every dose. In the fobrile cases the original dose was 1 two hundred thousandths milligiam T.R. The object was to work up to the strength of T.R. the patient would take without producing a reaction. When a reaction did occur the patient was kept quiet, and after a few days the same dose was repeatcd, and if no reaction followed the treatment was continued as before. If, how. ever, a reaction occurred a second time, then a further 
period of rest from tuberculin was given and was followed by a smaller dose than those which prodnced reactions. The maximum dose reached was one-tenth milligram, and this in the case of one patient only.

Method of Investigation.

We decided that the factors on which improvement or otherwise should be based must be two-namely:

1. The pulmonary condition as determined by the physical signs.

2. The general condition as determined by the weight, temperature, pulse-rate, cough, and amounts of sputum brought up daily by the patient.

These facts were all recorded daily about every patient in our series of cases, except the weight, which was taken fortnightly.

Special attention was paid to the physical signs. A systematic physical examination of every new patient was made and recorded without any information as to his history and without any reference to out-patient notes. A separate examination was made by the physician in charge of the case (Dr. Batty Sharv) in the same unbiassed way, and the two results were then compared. If any discrepancy appeared, an agreement was arrived at and the combined result recorded. Fortnightly examinations of the chest were made by one of us to note any gradual increase or decrease of signs, and prevent the fallacy of attributing a slight superimposed attack of bronchitis, etc., to the original condition. As it was noticed that after a day or two of rest in bed the physical signs tended to subside, the first examination for the comparison was not, made on the day of admission. This may not seem fair, as at the end of the three months the patient in many cases was doing exercise, but it must be remem bered that he had gradually worked up to this exercise and that he was doing no more than his condition was capable of; whereas several of the patients who had been working up to the time of admission had been doing more than their condition allowed and the lung was consequently very irritable. After rest of a day or two the lung in these cases had returned to what we might call its normal condition, and then a fair comparison could be made. A comparison of the relative increase or decrease of physical signs has been accepted as the only reliable clinical method of estimating the lung condition, but no one imagines the markings of physical signs to indicate the exact extent of the lesion, for post-nortem examination almost invariably proved the contrary.

At the end of three months each patient was examined separately by Dr. Batty Shaw and by one of us, and after a comparison of results an agreement was arrived at. The position of the apex beat and the mapping out of the cardiac dullness were found to be very important signs in the diagnosis of fibrnsis, and in more than one case a distinct alteration in the position of the heart, due to fibrosis of the affected lung, was noted.

ive must state further that when we report that a case showed an increase or decrease of signs at our final examination, it must be understood that the final estimate represents the end of a crescendo or decrescendo movement. Results.

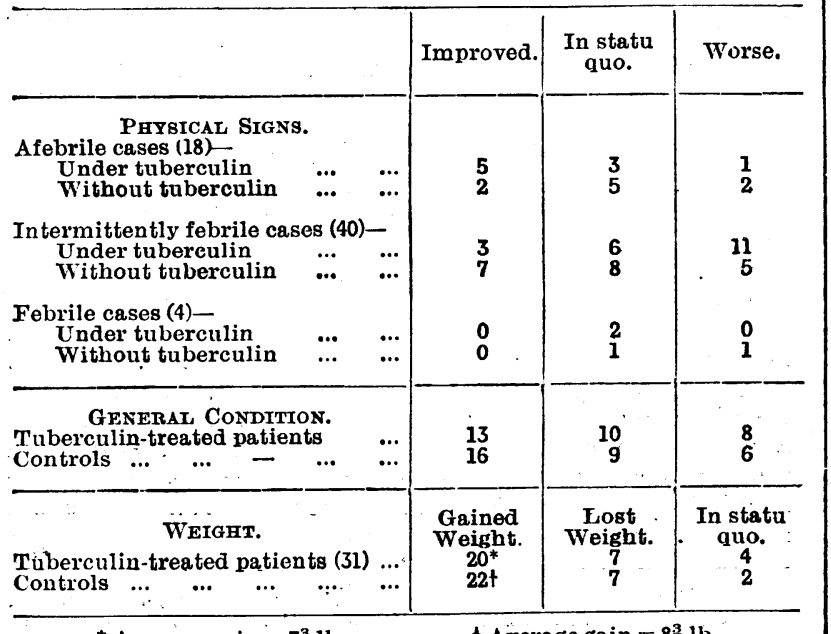

* Average gain $=7 x^{3} 1 \mathrm{~b}$.

t A verage gain $=8 \frac{3}{16}$
It will be noticed that in the table of results the weight statistics are recorded separately, though we have declared above our intention to include them under "General Con. dition." Further, it will be seen that the two records do not correspond, though we admit that the weight factor is an important one in estimating the general condition of a patient. The extreme difficulty of adding together all these varying factors-physical signs, weight, temperature, etc.-has-deterred us from publishing a summary of our results under one heading. The above statistics were com. pared in three sections quite independently of one another, and we conclude by drawing our inferences in the same independent manner.

Conclusions.

1. With regard to the local lesion, tuberculin seems to have done good in the afebrile cases, and though it will be pointed out, perhaps, that these are the only cases suitable for tuberculin, it must be remembered that such cases formed less than 30 per cent. of our total. Tuberculin was apparently harmful to the internittently febrile patients who formed the bulk of our series.

2. Tuberculin had no apparent effect in increasing the weight or in improving the general condition, and in both these cases the balance of improvement is on the side of the controls.

In conclusion we should-like to take this opportunity of thanking Dr. Batty Shaw for his kindness in 'allowing us to pursue these investigations among patients nndei his care.

\section{A NOTE ON SIXTY-THREE SUCCESSIVE CASES OF ENTERIC FEVER TREATED WITH VACCINES.}

BY W. P. MACARTHUR, M.D., F.R.C.P.IREL, Captain, R.A.M.C.

DURING the past year and a half I have been responsible for, the vaccine-therapy of 63 cases of typlioid fever. Although so small a series is of little statistical value its publication may interest those at least who wish to collect lists of such cases from as wide a field as possible, since this is the first series published from Mauritius.

From 61 of the 63 the causative organism was isolated, either from the blood (58) or from the faeces (3). All the cultures made from the two remaining cases were negative, but there is little doubt as to the diagnosis.

I have been accustomed to classify the cases as "favourable" and "unfavourable." The former class being those coming under vaccine treatment not later than the tenth day of the disease; and the latter those in which treatment was delayed until' the fourteenth day of the disease, or later. No doubt this division is somewhat arbitrary, but some such classification is necessary. It may be mentioned that the date of onset of any febrile infection can usually be determined much more readily here than at home, owing to a pronounced Mauritian weakness for taking temperatures on every possible occasion.

of the 63 cases under review, 45 belong to the "favourable" class, and 11 to the "unfavourable." They were average examples of the variety of enteric fever endemic in the colony, which resembles that found elsewhere. Some, however, believe that in Mauritius the disease tends to run a longer course than at home-possibly the septicaemic stage lasts longer-certainly, in my experience, the causative organism cain readily be isolated from the blood at a period of the disease when, according to home teaching, cultures are likely to be negative. Thus I was successful in isolating the bacillus from the blood in 12 out of 14 cases between the fourteenth and twenty-fourth days of the disease.

The age of the patients in this series raried from 18 months to 70 years, and they usually came under observation as undiagnosed cases with febrile symptoms. The routine procedure was then - an immediate blood culture. If the symptoms suggested possible enteric fever, an injection of a stock typhoid vaccine was given at the same time.

If the cultures showed B. typhosus, an autogenons vaccine was prepared without delay, with which the patient was treated subsequently. It appeared advisable 